\title{
Evolução clínica de pacientes submetidos à revascularização do miocárdio em um hospital universitário de 2004 a $2008^{1}$
}

\author{
Rita Inês Casagrande da Silva² \\ Leila Massaroni ${ }^{3}$ \\ Denise Silveira de Castro 4 \\ Eliana Zandonades \\ Maria Helena Costa Amorimº
}

doi:10.11144/Javeriana.ie18-1.ecps

Cómo citar: Silva RI, Massaroni. L, Castro, DS de, Zandonade E, Amorim MH. Evolução clínica de pacientes submetidos à revascularização do miocárdio em um hospital universitário de 2004 a 2008. Investig en Enferm. Imagem desarr. 2016;18(2):45-62. http:// dxdoi.org/1011144/Javeriana.ie18-1.ecps

1. Artigo de pesquisa original. Recebido: 21 de outubro de 2014. Aprobado: 17 de abril de 2015.

2. Mestre em Saúde Coletiva. Professora do Departamento de Enfermagem do Centro de Ciências da Saúde da Universidade Federal do Espírito Santo (UFES), Brasil. Doutoranda em Enfermagem pela Escola de Enfermagem Anna Nery da Universidade Federal do Rio de Janeiro, Brasil. Correio eletrônico: ritainesc@gmail.com

3. Doutora em Enfermagem. Professora do Departamento de Enfermagem do Centro de Ciências da Saúde da Universidade Federal do Espírito Santo (UFES), Brasil. Coordenadora adjunta do Programa de Pós Graduação em Enfermagem/UFES.

4. Doutora em Enfermagem. Professora do Programam de Pós Graduação em Enfermagem do Centro de Ciências da Saúde da Universidade Federal do Espírito Santo (UFES), Brasil.

5. Doutora em Estatística. Professora do programa de Pós Graduação em saúde Coletiva do Centro de Ciências da Saúde da Universidade Federal do Espírito Santo (UFES), Brasil.

6. Doutora em Enfermagem. Professora do Departamento de Enfermagem do Centro de Ciências da Saúde da Universidade Federal do Espírito Santo (UFES), Brasil. Coordenadora do Programa de Pós Graduação em Enfermagem/UFES. 


\section{Resumo}

Objetivo: Analisar a evolução clínica no período peri-operatório de pacientes submetidos à revascularização do miocárdio em hospital de ensino. Métodos: estudo descritivo de dados secundários de 147 prontuários de pacientes submetidos à revascularização do miocárdio (RM) em hospital de ensino, no período entre 2004 e 2008. Para a análise dos dados utilizou-se o método descritivo e o pacote estatístico para Ciências Sociais SPSS (18.0). Resultados: predominância do sexo masculino, com faixa etária entre 50 e 69 anos. A maioria dos pacientes, 66,7\% sofria de hipertensão arterial e 77,6\% tinham três ou mais doenças associadas. Tempo total de espera entre o cateterismo cardíaco e a RM apresentou mediana de 55 dias. A cirurgia foi suspensa em 26,53\% dos pacientes por problemas de estrutura, ao paciente e ao administrativo, sendo a falta de leito no centro de terapia intensiva (CTI) a principal causa. Constatou-se que $78,23 \%$ internaram na enfermaria. O tempo de permanência hospitalar teve mediana de 15 dias. O tempo de permanência no CTI teve mediana de 3 dias. Internação pré-operatória ocorreu uma vez em $21 \%$ dos pacientes. A reinternação ocorreu em $6,8 \%$ dos pacientes, tendo como principal motivo a infecção em 4,08\% dos pacientes. O óbito foi constatado em $11,6 \%$ dos pacientes com idade acima de 50 anos e a maioria, 76,5\% morreu no CTI. Conclusão: a análise das variáveis clínicas propostas apontou para resultados compativeis com o panorama nacional. Contudo, identificouse a fragilidade relacionada à gestão de recursos humanos e do desenvolvimento das atividades hospitalares, tendo como resultado, a fragmentação do atendimento e do planejamento assistencial.

Palauras chave: procedimentos cirúrgicos cardiacos; revascularização miocárdica; serviço hospitalar de cardiologia; assistência peri-operatória; acesso aos serviços de saúde

\section{Clinical Course of Patients Subject to Myocardial Revascularization Surgery in a University Hospital from 2004 to 2008}

\section{Abstract}

Objective: To analyze the clinical course in the perioperative phase of patients subject to myocardial revascularization surgery in a university hospital. Methods: Descriptive study of secondary data from 147 records of patients subject to myocardial revascularization surgery during the 2004-2008 period. A descriptive method was used along the SPSS Statistical Software Package (18.0). Results: Predominance of male patients, in the age group between 50 and 69 years. A $66.7 \%$ of them suffered arterial hypertension and $77.6 \%$ had 3 or more related diseases. The total waiting time between the cardiac catheterization and the surgery showed a median of 55 days. Surgery was suspended in $26.53 \%$ of the cases, due to structural problems, either patient or management-related. It was confirmed that a $78.23 \%$ of the patients was admitted to the infirmary and the total hospital stay time had a median of 15 days. The intensive care stay had a median of 3 days. Preoperative admittance happened once in a $21 \%$ of the patients. A new admittance happened in $6.8 \%$ of the patients, mainly due to infection (4.08\%). Conclusions: The analysis of the proposed clinical variables showed results coherent with the national outlook. However, we identified a weakness related to the management of human resources and the development of hospital activities. The result of the aforementioned weakness is the fragmentation of care and care planning.

Keywords: cardiac surgical procedures; myocardial revascularization; hospital cardiology service; perioperative care; accessibility of health services 


\section{Evolución clínica de pacientes sometidos a cirugía de revascularización miocárdica en un hospital universitario de 2004 a 2008}

\section{Resumen}

Objetivo: Analizar la evolución clínica en el periodo perioperatorio de pacientes sometidos a cirugía de revascularización miocárdica en un hospital universitario. Métodos: estudio descriptivo de datos secundarios de 147 registros de pacientes sometidos a cirugía de revascularización miocárdica en el periodo 2004-2008. Se utilizó el método descriptivo y el paquete estadístico SPSS (18.0). Resultados: Predominio del sexo masculino, con grupo etario entre 50 y 69 años. Un 66,7\% sufría de hipertensión arterial y el $77,6 \%$ tenía 3 o más enfermedades asociadas. El tiempo total de espera entre el cateterismo cardiaco y la cirugía presentó una mediana de 55 días. La cirugía fue suspendida en el $26,53 \%$ de los casos, por problemas de la estructura, con el paciente o administrativos. Se constató que el 78,23\% fue admitido por enfermería y el tiempo de estancia en el hospital tuvo una mediana de 15 días. La estancia en terapia intensiva tuvo una mediana de 3 de días. La internación preoperatoria ocurrió una vez en el $21 \%$ de los pacientes. Una nueva internación ocurrió en el 6,8\% de los pacientes, principalmente debido a la infección (4,08\%). Conclusión: El análisis de las variables clínicas propuestas señaló resultados coherentes con el panorama nacional. Sin embargo, se identificó la debilidad relacionada con la gestión de los recursos humanos y del desarrollo de las actividades hospitalarias, cuyo resultado es la fragmentación de la atención y de la planificación de la atención.

Palabras clave: procedimientos quirúrgicos cardiacos; revascularización miocárdica; servicio de cardiología en hospital; atención perioperativa; accesibilidad a los servicios de salud 


\section{Introdução}

Sob o ponto de vista epidemiológico, as condições crônicas de saúde estão aumentando em ritmo alarmante (1) e de acordo com o Ministério da Saúde de Brasil o estudo de indicadores relacionados às doenças crônicas não transmissiveis (DCNT) aponta que este grupo de doenças apresenta as maiores proporções, com $59 \%$ de anos de vida perdidos por morte prematura, $74,7 \%$ por anos de vida vividos com incapacidade e $66 \%$ por anos perdidos ajustados por incapacidade, sendo que neste grupo, os agravos cardiovasculares são as mais frequentes causas de morbi-mortalidade em nosso país, com elevado custo direto ou indireto, alto financiamento do sistema de saúde principalmente no nível de atenção da média e alta complexidade e para a reabilitação (2).

$\mathrm{O}$ atual cenário da saúde relacionado às doenças cardiovasculares difere substancialmente do início do século 20, onde as doenças cardiovasculares representavam menos de $10 \%$ das mortes no mundo. É esperado que o número de mortes por doenças cardiovasculares, principalmente doenças isquêmicas do coração e acidente vascular, alcance 23,3 milhões de pessoas em 2030 (1). Estas doenças representam até 50\% da mortalidade das DCNT e são as principais causas de internações e determinam o maior gasto do sistema de saúde nacional $(2,3)$.

Para o tratamento das doenças isquêmicas do coração a Sociedade Brasileira de Cardiologia reforça que a revascularização do miocárdio (RM) é uma importante intervenção terapêutica, devido ao seu impacto sobre os sintomas e o prognóstico. É amplamente indicada, de forma, em milhares de pacientes por sua comprovada redução de sintomas e aumento das taxas de sobrevivência, além de poder mudar a evolução natural da doença com reversão ou melhora dos sintomas clínicos, a qualidade e quantidade de vida (4).

A cirurgia cardiovascular é um tratamento complexo e exige vários fatores determinantes que impactam a realização deste procedimento, não dependendo apenas de habilidade técnica individual do médico para o sucesso. Embora seja uma rotina elaborada, precisa e mecanicista exige condições adequadas para a sua realização. Pesquisadores consideram que exige além da habilidade técnica individual do cirurgião, um conjunto de fatores são determinantes para o sucesso dessa assistência entre eles: fatores humanos, organizacionais institucionais, clínicos, estrutura de atendimento, socioeconômicos, financiamento reduzido, acesso difícil para a atenção básica (5-7).

Dessa forma torna-se necessário que a dinâmica hospitalar promova a agilidade na condução do paciente ao processo cirúrgico, diminuindo o impacto produzido pelas longas filas de espera, que levam por vezes a exacerbação do quadro clínico, elevação do número de óbitos por complicações pós-operatórias, demandando mais gastos para o setor saúde $(4,8)$.

Observam-se avanços tecnológicos, desde a descoberta de novas tecnologias, como técnicas mais modernas e de menores riscos para os tratamentos clínicos e cirúrgicos desses pacientes, sejam nas urgências ou no tratamento conservador $(6,7,9)$, todavia, o cotidiano hospitalar exibe uma complexidade organizacional que pode influenciar na assistência desses indivíduos (10).

Neste contexto a oferta de cuidados á saúde dos indivíduos que apresentam a doença em estágio avançado ou estado de agravamento, demanda 
a utilização dos métodos diagnósticos e terapêuticos especializados, tanto em nivel ambulatorial quanto hospitalar. Entretanto, os pacientes têm enfrentado vários desafios, tais como: o longo tempo de espera para consultas especializadas, para a realização de exames e procedimentos diagnósticos ou terapêuticos, bem como para a realização de internação. Diante do exposto objetivou-se com esse estudo: Analisar a evolução clínica no período perioperatório de pacientes submetidos à revascularização do miocárdio em um hospital de ensino.

\section{Método}

Trata-se de um estudo descritivo desenvolvido a partir da análise de dados secundários de prontuários de cirurgia de RM em um hospital de ensino, Vitória, Espírito Santo. A população constituiu-se por 179 prontuários de pacientes adultos que realizaram cirurgia de revascularização do miocárdio no período de 2004 a 2008.

Critérios de inclusão: prontuários de pacientes que passaram somente por cirurgia de RM nesse período e a ocorrência de reoperação até os primeiros os 30 dias.

Critérios de exclusão: cirurgia de prótese valvar associado à RM, prontuários incompletos, prontuários que não foram encontrados no serviço de arquivo no período da coleta.

A seleção da população deu-se por conveniência e por representar o percentual de procedimentos realizados ao longo dos últimos 10 anos de atendimento. Neste período obteve-se um total de 930 procedimentos, dentre estes 179 corresponderam a RM, dos quais 147 foram estudados e 32 excluídos, sendo oito prontuários devido á prótese valvar associado à RM, 11 não foram encontrados no serviço de arquivo e 13 prontuários estavam incompletos.

Compõem-se as variáveis do estudo em cinco grandes grupos: dados sociodemográficos: idade, sexo e procedência; dados pré-operatórios: tipo de doenças associadas a cardíaca, realização de exames pré-operatórios - hematócrito, creatinina sérica, ecocardiograma, cateterismo cardíaco (CATE), número de artérias obstruídas e o tempo decorrido entre o CATE e a RM-; dados do procedimento cirúrgico: tipo e quantidade de enxertos realizados, ocorrência e motivo provável de reoperação, suspensão do procedimento cirúrgico e o motivo; dados do fluxo intra hospitalar: tempo de internação total, tempo de permanência no Centro de Terapia Intensiva, ocorrência e motivo provável de óbito, internação pré-operatória, ocorrência de reinternação pós-operatória e o motivo; dados do fluxo ambulatorial: consulta pré-operatória, retorno ambulatorial pós operatório. Os dados foram colhidos pela pesquisadora com a utilização de um formulário para registros das variáveis estudadas contidas nos registros dos prontuários.

No tratamento estatístico realizou-se análise descritiva com tabela de frequência absoluta e relativa das variáveis, medianas, e desvio padrão para a análise dos tempos decorridos. Os dados foram inseridos na planilha do programa Microsoft Office Excel 2003 e analisou-os no Pacote Estatístico para Ciências Sociais (SPSS) versão (18.0). A pesquisa foi aprovada no Comitê de Ética do centro de Ciências da Saúde da Universidade Federal do Espírito Santo (registro $n^{\circ} 160 / 80$ ). 


\section{Resultados}

\section{Caracterização sociodemográfica}

A tabela 1 mostra predominância do sexo masculino, com faixa etária variando entre 35 e 81 anos, sendo a média de 60 anos. A RM ocorreu mais frequentemente na faixa etária entre os 50 e 69 anos com $62,6 \%$ dos pacientes. Os pacientes procederam na maioria dos municípios que compõe a Região Metropolitana de Vitória, com 78,2\%. Os demais municípios do interior do Estado, somados, representaram 25,9\% dos pacientes operados. Outros estados foram representados por Bahia e Minas Gerais, perfazendo $2,1 \%$ dos casos.

TABEla 1. Caracterização sócio-demográfica por sexo, idade e procedência no período de 2004 a 2008 no HUCAM-ES

\begin{tabular}{|c|c|c|}
\hline Variáveis & Número & Percentual \\
\hline \multicolumn{3}{|c|}{ Sexo } \\
\hline Masculino & 95 & 64,6 \\
\hline Feminino & 52 & 35,4 \\
\hline \multicolumn{3}{|c|}{ Faixa etária (anos) } \\
\hline Até 39 & 1 & 0,7 \\
\hline De 40 a 49 & 25 & 17,0 \\
\hline De 50 a 59 & 36 & 24,5 \\
\hline De 60 a 69 & 56 & 38,1 \\
\hline De 70 a 79 & 27 & 18,4 \\
\hline 80 ou mais & 2 & 1,4 \\
\hline \multicolumn{3}{|c|}{ Procedência } \\
\hline Cariacica & 33 & 22,4 \\
\hline Serra & 27 & 18,4 \\
\hline Vila Velha & 14 & 9,5 \\
\hline Vitória & 31 & 21,1 \\
\hline Interior do estado & 38 & 25,8 \\
\hline Outros estados & 3 & 2,1 \\
\hline Não informado & 1 & 0,7 \\
\hline Total & 147 & 100,0 \\
\hline
\end{tabular}

FonTE: elaboração própria com base nos dados do estudo de mestrado (11).

\section{Caracterização clínica pré-operatória}

Analisados conforme a tabela 2, dentre as doenças associadas $66,7 \%$ dos pacientes tinham hipertensão arterial e um total $77,6 \%$ dos pacientes tin-

50 ham de uma até três doenças associadas. A maioria dos resultados dos exames de hematócrito com $69,4 \%$, creatinina com $72,1 \%$ e CATE com $87,7 \%$, 
foram registrados no prontuário no pré-operatório. Já o ECO teve a minoria de informação no prontuário. Do total de CATE, 75,5\% ocorreram no período anterior à internação, em outros serviços, o qual foi denominado cateterismo ambulatorial. O cateterismo realizado após a internação correspondeu a $12,2 \%$ e foi denominado cateterismo intra-hospitalar. Em relação ao número de artérias obstruídas foi observado 29,3\% dos pacientes com três artérias obstruídas, 18,4\% com quatro obstruções. Em 12,9\% não foi encontrado informação no prontuário.

Tabela 2. Caracterização clínica por doenças associadas, tipos de exames, número de artérias obstruídas no periodo de 2004 a 2008 no HUCAM-ES. Continua

\begin{tabular}{|c|c|c|}
\hline Variáveis & Número & Percentual \\
\hline \multicolumn{3}{|c|}{ Doenças associadas $\dagger$} \\
\hline HAS & 98 & 66,7 \\
\hline DM & 36 & 24,5 \\
\hline Dislipidemia & 41 & 27,9 \\
\hline IAM & 37 & 25,2 \\
\hline Outras & 8 & 5,4 \\
\hline Sem doenças associadas & 16 & 10,9 \\
\hline Sem informação & 17 & 11,6 \\
\hline \multicolumn{3}{|c|}{ Número de doenças associadasłł } \\
\hline Uma & 38 & 25,9 \\
\hline Duas & 53 & 36,1 \\
\hline Três ou mais & 23 & 15,6 \\
\hline Sem doenças associadas & 16 & 10,9 \\
\hline Sem informação & 17 & 11,6 \\
\hline \multicolumn{3}{|c|}{ Tipo exames* } \\
\hline Hematócrito & 102 & 69,4 \\
\hline Creatinina & 106 & 72,1 \\
\hline Ecocardiograma & 58 & 39,4 \\
\hline Cateterismo cardíaco ambulatorial & 111 & 75,5 \\
\hline Cateterismo cardiaco intrahospitalar & 18 & 12,2 \\
\hline \multicolumn{3}{|c|}{ Número de artérias obstruídas | | } \\
\hline 1 & 14 & 9,5 \\
\hline 2 & 25 & 17,0 \\
\hline 3 & 43 & 29,3 \\
\hline 4 & 27 & 18,4 \\
\hline 5 & 16 & 10,9 \\
\hline 6 & 3 & 2,0 \\
\hline Sem informação & 19 & 12,9 \\
\hline
\end{tabular}

* Percentual calculado tomando como base os 147 casos.

$\dagger$ Total de doenças associadas $=253$.

Total de pacientes com doenças associadas $=147$.

$\S$ Total de exames realizados $=395$.

|| total de pacientes com número de artérias obstruídas $=147$.

Fonte: elaboração própria com base nos dados do estudo de mestrado (11). 
A tabela 3 descreve a variação de zero a 466 dias entre o CATE e a cirurgia com mediana de 55 dias e desvio-padrão de 109,99 dias de espera. Aponta que o CATE ambulatorial obteve a maior variação de zero a 466 dias com mediana de 60,50 dias e desvio-padrão de 112,06 dias de espera para a RM. O tempo dos que foram a óbito foi ainda maior com variação de 1 a 466 dias e mediana de 106 dias.

TABela 3. Caracterização de dados pré-operatórios por tempo decorrido entre o CATE até a RM no período de 2004 a 2008 no HUCAM-ES

\begin{tabular}{|l|c|c|c|c|c|}
\hline \multicolumn{1}{|c|}{ Tempo (em dias) } & Média & Mediana & $\begin{array}{c}\text { Desvio- } \\
\text { padrão }\end{array}$ & Minimo & Máximo \\
\hline $\begin{array}{l}\text { Entre o cateterismo e } \\
\text { a cirurgia }\end{array}$ & 97,22 & 55,00 & 109,99 & 0 & 466 \\
\hline $\begin{array}{l}\text { Entre o cateteris- } \\
\text { mo ambulatorial e a } \\
\text { cirurgia }\end{array}$ & 106,72 & 60,50 & 112,06 & 0 & 466 \\
\hline $\begin{array}{l}\text { Entre o cateterismo } \\
\text { intra hospitalar e a } \\
\text { cirurgia }\end{array}$ & 12,54 & 12,00 & 8,17 & 1 & 33 \\
\hline $\begin{array}{l}\text { Entre o cateterismo } \\
\text { intra hospitalar e } \\
\text { internação }\end{array}$ & 4,57 & 3,50 & 4,03 & 1 & 466 \\
\hline $\begin{array}{l}\text { Tempo entre cateteris- } \\
\text { mo e cirurgia (dos que } \\
\text { foram a óbito) }\end{array}$ & 140,38 & 106,00 & 153,51 & 1 & \\
\hline
\end{tabular}

FONTE: elaboração própria com base nos dados do estudo de mestrado (11).

\section{Caracterização do procedimento cirúrgico}

Observa-se na tabela 4 que houve variação entre a utilização de um a seis enxertos, sendo que o maior número de pacientes $89,2 \%$ receberam entre dois a quatro enxertos, totalizando 435 pontes, tendo como a média 2,95 enxertos para cada paciente. Em relação ao tipo de enxerto, em $72,1 \%$ foi usado a safena e a mamária. Do total investigado $4,76 \%$ pacientes foram submetidos à reoperação, tendo ocorrido em sua maioria no transoperatório ou durante os primeiros trinta dias. Destes, a reoperação teve o maior percentual relacionado à mediastinite com $2,72 \%$ dos casos.

O procedimento cirúrgico foi suspenso em $26,53 \%$ dos pacientes. Desses, $18,37 \%$ o tiveram suspenso uma vez, $6,12 \%$ e para um paciente este procedimento suspenso quatro vezes conforme tabela 5 . A suspensão ocorreu por 58 motivos, sendo que desses o maior percentual, 60,3\% (35) por problemas lacionados ao paciente e $15,5 \%$ (9) relacionadas às questões administrativas. 
TABela 4. Caracterização do procedimento cirúrgico por número e tipo de enxerto, número de artérias obstruídas, reoperação e suspensão de cirurgia no período de 2004 a 2008 no HUCAM-ES

\begin{tabular}{|c|c|c|}
\hline Variáveis & Número & Percentual \\
\hline \multicolumn{3}{|c|}{ Número de enxertos } \\
\hline 1 & 10 & 6,80 \\
\hline 2 & 38 & 25,90 \\
\hline 3 & 55 & 37,40 \\
\hline 4 & 38 & 25,90 \\
\hline 5 & 4 & 2,70 \\
\hline 6 & 2 & 1,40 \\
\hline \multicolumn{3}{|c|}{ Tipos de enxertos } \\
\hline Safena & 28 & 19,00 \\
\hline Mamária & 10 & 6,80 \\
\hline Safena + mamária & 106 & 72,10 \\
\hline Safena + mamária + radial & 2 & 1,40 \\
\hline Outras & 1 & 0,70 \\
\hline \multicolumn{3}{|c|}{ Motivos de reoperação* } \\
\hline Mediastinite & 4 & 2,72 \\
\hline Hemorragia & 2 & 1,36 \\
\hline Tamponamento & 1 & 0,68 \\
\hline Não reoperaram & 140 & 95,24 \\
\hline \multicolumn{3}{|c|}{ Pacientes com cirurgias suspensas* } \\
\hline Não & 108 & 73,47 \\
\hline Sim & 39 & 26,53 \\
\hline Total & 147 & 100,00 \\
\hline
\end{tabular}

* Percentual calculado tomando como base os 147 pacientes.

FonTE: elaboração própria com base nos dados do estudo de mestrado (11).

TABela 5. Caracterização de dados do procedimento cirúrgico por ocorrência de suspensão de cirurgia no período de 2004 a 2008 no HUCAM-ES

\begin{tabular}{|l|c|c|c|}
\hline \multicolumn{1}{|c|}{$\begin{array}{c}\text { Pacientes com cirurgias } \\
\text { suspensas }\end{array}$} & $\begin{array}{c}\text { Total de } \\
\text { pacientes }\end{array}$ & Percentual* & $\begin{array}{c}\text { Total de } \\
\text { cirurgias } \\
\text { suspensas }\end{array}$ \\
\hline Não & 108 & 73,47 & $\ldots$ \\
\hline Sim & 39 & 26,53 & $\ldots$ \\
\hline Uma vez & 27 & 18,37 & 27 \\
\hline Duas vezes & 9 & 6,12 & 18 \\
\hline Três vezes & 2 & 1,36 & 6 \\
\hline Quatro vezes & 1 & 0,68 & 1 \\
\hline Total & 147 & 100,00 & 52 \\
\hline
\end{tabular}

* Percentual calculado tomando como base os 147 casos.

FonTe: elaboração própria com base nos dados do estudo de mestrado (11). 


\section{Caracterização do fluxo intra hospitalar}

Observa-se na tabela 6 que o maior percentual de internação foi na Enfermaria com $78,23 \%$ dos pacientes O tempo de permanência hospitalar total variou de 6 a 108 dias, mediana de 15 dias. Já o tempo de permanência no CTI variou de um a 46 dias, mediana de 3 dias. A internação pré-operatória ocorreu pelo menos uma vez em $21 \%$ dos pacientes e no total $31,3 \%$ de pacientes que tiveram alguma internação. Já a reinternação em apenas 6,8\% dos pacientes, tendo como principal motivos a infecção com $4,08 \%$ dos pacientes. O óbito foi constatado em $11,6 \%$ dos pacientes, tendo ocorrido com idade acima de 50 anos e a maioria $76,5 \%$ morreram no CTI.

Tabela 6. Caracterização do fluxo intra-hospitalar, por local de internação número de internação pré-operatórias, reinternação, motivo da reinternação, número e local do óbito no período de 2004 a 2008 no HUCAM-ES

\begin{tabular}{|c|c|c|}
\hline Local da internação & Número & Percentual \\
\hline Enfermaria & 115 & 78,2 \\
\hline os & 23 & 15,6 \\
\hline CTI & 5 & 3,4 \\
\hline Hemodinâmica & 2 & 1,4 \\
\hline Centro cirúrgico & 2 & 1,4 \\
\hline \multicolumn{3}{|c|}{ Número de internações pré-operatórias realizadas } \\
\hline Nenhuma & 101 & 68,7 \\
\hline Uma & 31 & 21 \\
\hline Duas & 6 & 4,1 \\
\hline Três & 7 & 4,8 \\
\hline Quatro & 2 & 1,4 \\
\hline \multicolumn{3}{|c|}{ Ocorrência de reinternação pós-operatória } \\
\hline $\mathrm{Sim}$ & 10 & 6,8 \\
\hline Não & 137 & 93,2 \\
\hline \multicolumn{3}{|c|}{ Motivos de reinternação* } \\
\hline Infecção & 6 & 4,08 \\
\hline Dor torácica & 2 & 1,36 \\
\hline Causas não cardíacas & 1 & 0,68 \\
\hline ICC descompensada & 1 & 0,68 \\
\hline Não reinternaram & 137 & 93,2 \\
\hline \multicolumn{3}{|c|}{ Número de óbito } \\
\hline Sim & 17 & 11,6 \\
\hline Não & 130 & 88,4 \\
\hline \multicolumn{3}{|c|}{ Local do óbito } \\
\hline CTI & 13 & 76,5 \\
\hline $\mathrm{CC}$ & 4 & 23,5 \\
\hline Total & 147 & 100,0 \\
\hline
\end{tabular}

FONTE: elaboração própria com base nos dados do estudo de mestrado (11). 


\section{Caracterização de dados do fluxo ambulatorial}

Houve variação de uma a cinco consultas pré-operatória, sendo que 13,6\% tiveram uma consulta e $72 \%$ não foi identificado registros desse atendimento. Do total de casos apenas $32,7 \%$ efetuaram o retorno ambulatorial pósoperatório, tendo uma variação de $14,3 \%$ com um retorno e $0,7 \%$ retornaram sete e doze vezes cada.

\section{Discussão}

\section{Caracterização sociodemográfica}

Idade. O perfil da população estudada reflete semelhança com o resultado de outros estudos realizados no Brasil. Em geral os estudos nesta área apontam a idade como um determinante crítico para complicações e mortalidade após a cirurgia de RM. Houve predominância do sexo masculino estando de acordo com o encontrado em resultados de pesquisas de outros autores (12). Na maioria das faixas etárias, o risco de doenças cardiovasculares é mais elevado no sexo masculino, embora esta diferença diminua com o avanço da idade $(6,12,13)$.

Procedência. O estudo da procedência do paciente é importância, pois retrata o deslocamento da população interiorana em busca de assistência em regiões central, refletindo capacidade resolutiva dos Municípios cujo padrão de financiamento e suporte tecnológico e de capacitação dos profissionais não oferecem recursos capaz de suprir a necessidade de assistência de maior complexidade. Este fator tem implicação direta no financiamento e planejamento de recursos estruturais e número de leitos eficazes de acordo com a capacidade instalada disponível no sistema de saude brasileiro. Essa situação ainda é comum em São Paulo, pois ainda interna 15\% de cidadãos de outras cidades (14).

\section{Caracterização clínica pré-operatória}

Doenças associadas. A maioria dos pacientes tinha de uma a duas doenças associadas, dentre elas, a HAS foi a mais frequente, seguida da dislipidemia, IAM prévio e diabetes. Essas doenças são as mais investigadas neste universo, por serem, ao mesmo tempo, fator de risco para as DCV como para a realização da RM. A Sociedade Brasileira de Cardiologia orienta a realização da investigação pré-operatória para a identificação da presença de outras comorbidades associadas à Insuficiência coronariana (IC), com a finalidade de minimizar os riscos cirúrgicos e prevenir as complicações pós-operatórias. A presença de doenças associadas à doença cardíaca pode influenciar na decisão sobre o momento adequado para a realização da cirurgia $(4,8)$. Pesquisadores corroboram com essa afirmação e investigam em estudos, o perfil clínico da população como a presença de diabetes melittus, hipertensão, doença pulmonar obstrutiva crônica (DPOC), insuficiência renal, dislipidemia, entre outros fatores (12). 
Exames pré-operatórios. Todos os exames pesquisados compõem o protocolo de pré-operatório, são recomendados pela SBC, pois oferecem informações clínicas importantes sobre metabolismo celular, alterações anatômicas e funcionais do organismo humano, assim como anormalidades que possam interferir na decisão cirúrgica e oferecer riscos de intercorrências peri-operatórias $(4,8)$.

Número de artérias obstruídas. O número de artérias obstruídas indica um dos aspectos de gravidade da DAC, sendo relevante para a caracterização do paciente coronariano cirúrgico, pois exibe a extensão da doença e com isso o perfil da clientela atendida por um determinado serviço (4). Podese observar no estudo atual que o maior número de pacientes apresentou obstruções de duas a quatro artérias, perfazendo um total de $64,7 \%$ evidenciando um perfil de outros estudos (12).

Tempo decorrido entre o CATE e a RM. Especialistas afirmam que "tratamentos cirúrgicos quando indicados para os portadores de doença coronariana isquêmica, apresentam melhores resultados se ofertados em tempo apropriado, sem demoras desnecessárias" (10). Outros ressaltam que ainda não é comum encontrar na literatura médica dados disponíveis sobre o tempo entre a marcação e a realização de cirurgia de revascularização miocárdica eletiva (15). Dados de estudo da Suécia apontaram que o tempo médio de espera total foi de 55 dias com uma variação de 21 a 105 dias. Ao agrupar por critérios, os pacientes que foram classificados como emergência aguardaram uma média de 17 dias, sendo a variação de 7 a 39 dias.

Os autores consideram que as listas de espera para procedimentos médicos são bastante comuns em países que proporcionam acesso universal ao sistema de saúde. Para procedimentos, como cirurgia de revascularização do miocárdio, a espera prolongada associa-se a um risco aumentado de complicações graves, muitas vezes fatais. O conhecimento de fatores preditivos de complicações durante a espera pode ajudar a aperfeiçoar a forma de priorização e reduzir a chance de eventos no período (16), confirmando a importância do estudo desta variável. Neste estudo foi avaliado o tempo de espera do CATE até a RM, contudo é desconhecida a lista de espera existente neste hospital e as possiveis intercorrências com esses pacientes. Embora a avaliação inicial do tempo de espera deste estudo esteja dentro, ou até melhor em número de dias de espera para a realidade nacional e internacional para serviços públicos, não podemos comparar, pois as realidades e as variáveis não podem ser alinhadas, oferecendo-nos apenas um panorama desta e de outras realidades, bem como das possibilidades de eventos intercorrentes importantes para os pacientes e familiares enquanto aguardam a cirurgia de RM. O conhecimento desta realidade poderá direcionar o planejamento da assistência ofertada pelas Instituições, bem como para os gestores públicos de saúde.

\section{Caracterização do procedimento cirúrgico}

Número de enxertos. As diretrizes da doença coronariana crônica recomendadas pela Sociedade Brasileira de Cardiologia considera que algumas 
variáveis intra-operatórias entre elas o tempo prolongado de CEC e o número de enxertos podem ser fatores de risco para a ocorrência de Acidente vascular cerebral no periodo peri-operatório (8). Os achados deste estudo exibem um perfil compatível com outros estudos realizados no Brasil $(4,12,17)$.

Tipos de enxertos. O padrão ouro na cirurgia de RM é a utilização da artéria torácica interna esquerda (ATIE) por apresentar vantagens sobre os demais enxertos sobre a mortalidade e perveabilidade do enxerto. Afirma ainda que a utilização exclusiva de enxertos arteriais, especialmente a dupla mamária pode ser a melhor opção para o tratamento em longo prazo reduzindo o índice de falência dos condutos e os resultados tardios $(4,7,18)$. Existem muitas discussões na literatura sobre o tipo de enxerto para as variadas aplicações, contudo o uso do enxerto venoso parecer ser convencional nas condições de emergência pela maior rapidez no preparo do que a artéria mamária (9).

Ocorrência de reoperação. Foi identificado a ocorrência de reoperação com o total de 4,76\%, tanto no pós-operatório imediato como no tardio. Esteve relacionada a mediastinite, sangramento e tamponamento cardíaco. Outro estudo identificou um percentual de $27,2 \%$ de reoperação em sua maioria por hemostasia (19). Neste estudo foi considerado reoperação o período imediato de pós-operatório até trinta dias após a cirurgia de RM. Os casos de hemorragia e tamponamento cardíaco foram em condições imediatas e os casos de mediastinite foram durante a internação, tendo apenas um caso de retorno ambulatorial para desbridamento cirúrgico no esterno nos primeiros trinta dias.

Suspensão da cirurgia. A análise desta variável pode ser de grande relevância institucional, pois aponta para ações organizacionais tanto nos aspectos relativos à estrutura como aos processos de trabalho e práticas interdisciplinares. Neste estudo a RM foi suspensa por vários motivos os quais foram agrupados em três grupos: estrutura, paciente e administrativo. Os motivos relacionados à estrutura foram responsáveis por $60,3 \%$ das suspensões do procedimento. Desses, a falta de leitos de CTI foi o mais frequente com $80 \%$ das suspensões, a falta de equipamentos 14 , 3\% e a falta de hemoderivados foi de $5,7 \%$. Os motivos relacionados aos pacientes representaram $24,2 \%$, sendo que a maioria deles, exceto o paciente que faltou a cirurgia, poderia ter a suspensão evitada com ações de orientação pré-operatória e cuidados adequados. Os motivos administrativos que impediram a cirurgia somaram 15,5\%. Esses motivos foram separados da estrutura, pois houve uma percepção de que embora estejam ligados à gestão, estes motivos apontam para ações dos profissionais e ao trabalho multidisciplinar e multidimensional da atenção no perioperatório, especialmente relacionados à comunicação entre os diversos profissionais e diversos setores por onde o paciente cirúrgico circula. A suspensão cirúrgica gera diversos desdobramentos como: alteração do planejamento do mapa cirúrgico, comprometimento na produção e faturamento do hospital, estresse dos profissionais e principalmente os sentimentos de insegurança e muitas vezes até de desconfiança dos pacientes sobre os cuidados prestados. Nessas situações observa-se que a frustração atinge tanto ao paciente como as equipes de saúde, pois de modo 
geral todos os eventos que causaram a suspensão de cirurgias relacionados aos pacientes e os administrativos, em sua maioria, poderiam ter sido evitadas mediante as ações de um programa multidisciplinar de atendimento pré-operatório. Reflete também o sucateamento de hospitais, especialmente os federais, observado ao longo das últimas décadas o qual ocasiona uma problemática administrativa, gerencial, com falta de recurso tecnológico, recursos humanos capacitados, número de leitos disponiveis para a assistência intensiva $(5,15)$.

Local de internação. O maior contingente de cirurgiados $(78,2 \%)$ internaram na Enfermaria. O local da internação dos pacientes não tem sido avaliado por pesquisadores, contudo a avaliação do fluxo dos pacientes tem grande relevância para um hospital de ensino com grandes gargalos, com relação à oferta de serviços pela alta demanda de leitos e procedimentos de alta complexidade como a cirurgia de revascularização do miocárdio. Esse percentual esteve próximo de outro estudo, entretanto a maioria foi proveniente da Unidade de emergência (20).

Tempo de internação total. A análise desta variável é de extrema relevância, pois reflete o estado clínico dos pacientes, as complicações pós-operatórias, dependendo do diagnóstico principal e associados, procedimento principal, entre outros procedimentos operatórios, idade do paciente e condições de alta hospitalar (21). O presente estudo obteve tempo de internação total e tempo de internação no CTI aproximados ao de outros estudos realizados $(19,22)$. É provável, que nesse estudo, isto se deva ao maior tempo de permanência daqueles que apresentaram complicações no pós-operatório, assim como por suspensão de cirurgias em que o paciente não recebeu alta, tanto pelas condições clínicas, como pelas condições sociais. Outros pesquisadores consideraram que a variação no tempo de internação pode estar relacionada com o número de pontes implantada, sendo maior naqueles que receberam de três a cinco pontes (15). O Tempo de permanência no CTI após a cirurgia correspondeu à admissão no CTI após a RM até a alta do mesmo para o setor de internação e apresentou resultado aproximado ao de outros estudos $(19,22)$.

Ocorrência de óbito. A incidência de mortalidade tem sido muito estudada tanto em pesquisas clínicas, como em base de dados secundários na área cardiológica pelos riscos de morte que os portadores de doença coronariana estão sujeitos. Ainda, tem sido utilizada como indicador de qualidade de serviços e de capacidade técnica do cirurgião, contudo há uma expectativa de que o uso desse indicador seja pautado em modelos de predição de risco cirúrgico para que os índices possam ser comparados e a qualidade tenha uma lógica para sua aferição, pois os pacientes indicados para RM diferem muito em extensão da doença coronariana e complicações clínicas, bem como pela associação de outras doenças $(5,7,18,23-25)$. A taxa de óbito no presente estudo está em acordo com outros estudos (22), tendo a maioria ocorrido no CTI e a seguir no Centro Cirúrgico. O motivo mais frequente de suspeita de causar o óbito foi o estado de choque (vários tipos de choque) com $52,9 \%$. Outros pesquisadores encontraram outras prováveis causas como $2,2 \%$ relacionados a causas cardiológicas, $0,49 \%$ por infecção generalizada, 
$0,24 \%$ por insuficiência renal, $0,74 \%$ por broncopneumonia, $0,24 \%$ por causa metabólica, sangramento e acidente vascular cerebral respectivamente (12). Ocorrência de internação pré-operatória. Observado escassez de estudos desta variável em literatura científica, entretanto o estudo da mesma poderá ser relevante para compreender questões relacionadas ao fluxo intrahospitalar do paciente, tendo em vista que os pacientes cirúrgicos internam outras vezes, antes da realização da RM por desequilíbrio do quadro de saúde enquanto aguardam as filas de espera. A internação pré-operatória pode ser oportuna para que ele e sua família conheçam a equipe de saúde e o ambiente hospitalar. O que poderá favorecer o enfrentamento emocional de medo, ansiedade, angústia e preocupação com o procedimento cirúrgico (26).

Ocorrência de reinternação pós-operatória. Os motivos para a reinternação foram descritas como registradas nos prontuários e foram agrupados por: infecção, dor torácica, causas não cardíacas e ICC descompensada. Para o grupo de infecção foram considerados as causa como: "abscesso de parede do esterno", "pneumonia", "infecção da ferida operatória no Membro Inferior", "infecção da ferida cirúrgica - mediastinite", "ferida operatória em MID infectada", "infecção em ferida cirúrgica". Para o grupo de dor torácica foram considerados: "dor precordial" e "dor torácica", causas não cardíacas relacionada a "epigastralgia" e por último como causa individual a "ICC descompensada", conforme registros em evolução clinica dos prontuários. A reinternação pode ocorrer por complicações da cirurgia ou por novos eventos cardiacos (27). A taxa observada em pesquisa em hospital privado foi menor com $2,1 \%$ e esteve relacionada a infecção em ferida cirúrgica (15) e tem sido utilizada como um indicador de qualidade desse serviço hospitalar $(21,28)$.

Consulta pré-operatória ambulatorial. A consulta pré-operatória é de extrema importância para avaliação clínica, orientação diagnóstica, terapêutica e para a identificação dos cuidados á saúde. O ideal é que seja realizada por uma equipe multiprofissional, pois a complexidade da demanda de cuidado desse paciente e sua família exigem medidas de integração entre os profissionais, pacientes e familiares. A dimensão do vínculo emocional e terapêutico que pode ser criado nos ambientes de consultas poderá contribuir para que os pacientes e familiares vençam os medos e as inseguranças comuns no enfrentamento desse paciente frente a esse evento cirúrgico (26). Neste estudo não foi encontrado registro de realização de consulta pré-operatória na maioria dos prontuários. Este dado pode refletir a necessidade de intervenção tanto em nivel de gestão para a adequação de condições para a realização dos registros, para a realização das consultas pré-operatórias, bem como e a adoção de medidas assistenciais multidisciplinares para o atendimento dos pacientes coronarianos e para o preparo pré-operatório dos mesmos.

Retorno pós-operatório ambulatorial. Não foi encontrado registros de retorno ambulatorial na maioria dos prontuários. Esses dados sugerem que os pacientes em sua maioria procedentes dos municípios da Região Metropolitana da Grande Vitória não retornaram ao serviço para a avaliação pós-operatória de RM. O oposto foi demonstrado por outros pesquisadores em que os pacientes realizaram, em média, quatro consultas no período 
estudado, sendo uma consulta a cada dois meses e pode estar associada à elevada frequência do uso de fármacos no pós-operatório sem um controle satisfatório (12). O acompanhamento sistematizado é um desafio profissional e institucional em que o maior benefício poderá ser o do indivíduo através de uma assistência integral e de qualidade e da instituição por oferecer segurança e metodologia para esse nível de atendimento.

\section{Conclusão}

De modo geral, tanto as variáveis estudadas nos cincos grupos propostos como os resultados são compatíveis com outros estudos nacionais. Alguns pontos chamam a atenção nesse cenário, como a ausência de registros de dados da história de paciente e de resultados de exames nos prontuários; suspensão de procedimento cirúrgico por motivos relacionados à estrutura, ressaltando a ausência de investimento nos hospitais federais nas últimas décadas; suspensão do procedimento cirúrgico por motivos relacionados ao paciente e os administrativos que somados ao número reduzido de consultas pré e pós-operatória, denota a fragilidade de questões relacionadas à gestão de recursos humanos, assim como a fragmentação de atividades relacionadas ao planejamento assistencial. Observado também que o estudo de variáveis como suspensão de cirurgia e do fluxo de pacientes ambulatorial e intra-hospitalar ainda são escassos. As autoras acreditam que a adoção de medidas estratégicas visando a melhoria do processo de trabalho e de tecologia para a gerência e para a efetuação de registros em prontuário; a adoção de estatégia interdisciplinar no cuidado ao paciente no atendimento clínico, cirúrgico, intra e extra-hospitalar. Consideram importante ainda a criação de protocolos assistenciais de avaliação de risco, que possam auxiliar as equipes na assistência especializada. Sugerem que a implementação de um banco de dados para sistematizar coleta de informações possa auxiliar as ações de gerência e para a tomada de decisão referentes ao atendimento cirúrgico dos pacientes revascularizados do miocárdio, bem como pode apoiar comparações no próprio serviço e entre instituições, favorecendo também a pesquisa nesta área.

\section{Conflito de interesses}

As autoras declaram não houver conflitos de interesses pertinentes.

\section{Financiamento}

E o presente estudo não teve fontes de financiamento externas.

\section{Referências}

1. World Health Organization. Cardiovascular diseases [internet]; 2013 [citado 2013 dez 18]. Disponivel em http://www.who.int/ cardiovascular_diseases/en/.

2. Ministério da Saúde (BR); Organização Pan-Americana da Saúde. Doenças crônicas não transmissiveis: estratégias de controle e desafios e para os sistemas de saúde [internet]. Brasília; 2011 [citado 2015 mar 15]. Disponivel em: http://apsredes.org/site2012/wp-content/ uploads/2012/06/Condicoes-Cronicas_flavio1.pdf. 
3. Ministério da Saúde (BR). Plano de ações estratégicas para o enfrentamento das doenças crônicas não transmissiveis (DCNT) no Brasil 2011-2020 [internet]. Brasília; 2011 [citado 2015 mar 15]. Disponível em: http://actbr.org.br/uploads/conteudo/918_cartilha_dent.pdf.

4. Cesar LA, Ferreira JF, Armaganijan D, Gowdak LH, Mansur AP, Bodanese LC, et al. Diretriz de doença coronária estável. Arq Bras Cardiol. 2014;103(2 Supl 2):1-59.

5. Gomes WJ, Mendonça JT, Braile DM. Resultados em cirurgia cardiovascular: oportunidade para rediscutir o atendimento médico e cardiológico no sistema público de saúde do país [editorial]. Rev Bras Cir Cardiovasc. 2007;22(4):III-VI.

6. Carvalho MRM, Silva NAS, Oliveira GMM, Klein CH. Associação de fatores pré-operatórios e óbitos na cirurgia de revascularização miocárdica em hospitais públicos do Rio de Janeiro: 1999-2003. Rev SOCERJ. 2008;21(5):311-9.

7. European Society of Cardiology (ESC); European Association of CardioThoracic Surgery (EACTS). Recomendações sobre revascularização do miocárdio. Eur Heart J. 2011;30:951-1005.

8. Sociedade Brasileira de Cardiologia (SBC). Diretrizes da Sociedade Brasileira de Cardiologia sobre angina instável e infarto agudo do miocárdio sem supradesnivel do segmento ST. Arq Bras Cardiol. 2014;102(3Supl. 1):1-61.

9. Carvalho ARS. Estudo retrospectivo das complicações intraoperatórias na cirurgia de revascularização do miocárdio. Rev Eletrônica Enferm [internet]. 2009;10(4):1057-65. Disponivel em: http://www.fen.ufg.br/ revista/v10/n4/v10n4a18.htm.

10. Murad H, Murad FF. Controle de qualidade em cirurgia cardiovascular: um paradigma a ser atingido. Rev Bras Cir Cardiovasc. 2007;22(4):470-5.

11. Silva, RIC. Perfil dos pacientes revascularizados do miocárdio no período de 2004 a 2008 em um Hospital Universitário do Espírito Santo [dissertação de mestrado]. Universidade Federal do Espírito Santo. Centro de ciências da Saúde, Programa de Pós-Graduação em Saúde Coletiva, Vitória; 2009.

12. Barbosa ACC, Passos LCS, Lopes AA, Andrade WLG, Souza LR, Nunes MB. Avaliação da adequação do controle de fatores de risco cardiovascular após cirurgia de revascularização miocárdica. Arq bras cardiol. 2007;89(6): 362-9.

13. Milani R, Milani R, Brofman P, Moutinho JA, Barboza L, Guimarães $\mathrm{M}$, Barbosa A, et al. Revascularização total do miocárdio sem circulação extracorpórea em pacientes com disfunção ventricular esquerda. Arq bras cardiol. 2007;89(1):11-5.

14. Vecina G Neto, Malik AM. Tendências na assistência hospitalar. Ciênc Saúde Coletiva. 2007;12(4):825-39.

15. Mesquita ET, Ribeiro A, Araújo MP, Campos LAAC, Fernandes MA, Colafranceschi AS, et al. Qualidade assistencial em revascularização miocárdica. Arq Bras Cardiol. 2008;90(5):350-4. 
16. Rexius H, Brandrup-Wognsen G, Odén A, Jeppsson A. waiting time and mortality after elective coronary artery bypass grafting. Ann Thorac Surg. 2005;79:538-43.

17. Peixoto RS, Pires Jr HR, Netto MRS, Montes Pena FM, Areas GS, Moraes FVD, et al. Revascularização miocárdica no idoso: experiência de 107 casos. Rev SOCERJ. 2009;22(1):24-30.

18. American College of Cardiology Foundation (ACCF); American Heart Association (AHA). Guideline for coronary artery bypass graft surgery: A report of the American College of Cardiology Foundation/American Heart Association task force on practice guidelines [internet]; 2011 [citado 2015 mar 15]. Disponivel em: http://content.onlinejacc.org/ article.aspx?articleid $=1147818$.

19. Anderson AJPG, Barros Neto FXR, Costa MA, Dantas LD, Hueb AC, Prata MF. Preditores de mortalidade em pacientes acima de 70 anos na revascularização miocárdica ou troca valvar com circulação extracorpórea. Rev Bras Cir Cardiovasc. 2011;26(1):69-75.

20. Campagnucci PV, Silva AMRP, Pereira WL, Chamlian EG, Gandra SMA, Rivetti LA. EuroSCORE e os pacientes submetidos a revascularização do miocárdio na Santa Casa de São Paulo. Rev Bras Cir Cardiovasc. 2008;23(2):262-7.

21. Escrivão A Jr. Uso da informação na gestão de hospitais públicos. Ciênc Saúde Coletiva. 2007;12(3):655-66.

22. Araujo NR, Araújo RA, Oliveira RC, Bezerra SMMS. Complicações pósoperatórias em pacientes submetidos a cirurgia de revascularização miocárdica. Rev Enferm UFPE. 2013;7(5):1301-10.

23. Santos CA, Oliveira MAB, Brandi AC, Botelho PHH, Brandi JCM, Santos MA, et al. Risk factors for mortality of patients undergoing coronary artery bypass graft surgery. Rev Bras Cir Cardiovasc. 2014;29(4):513-20.

24. Ramos ARWR, Flores MB, Libonati RM, Quaresma AS. Preditores de mortalidade na cirurgia de revascularização do micáridio. Rev Bras Cardiol. 2013;26(3):193-9.

25. Giffhorn H. Avaliação de uma escala de risco em pacientes submetidos a cirurgia de revascularização do miocárdio: análise de 400 casos. Rev Bras Ter Intensiva [internet]. 2008 [citado 2015 mar 15];20(1):6-17. Disponivel em: http://dx.doi.org/10.1590/S0103-507X2008000100002.

26. Strabelli TMV, Stolf NAG, Uip DE. Uso prático de um índice de risco de complicações após cirurgia cardíaca. Arq Bras Cardiol. 2008;91(5):342-7.

27. Nery RM, Barbisan JN, Mahmud MI. Influência da prática da atividade física no resultado da cirurgia de revascularização miocárdica. Rev Bras Cir Cardiovasc. 2007;22(3):297-302.

28. Oliveira EL, Westphal GA, Mastroeni MF. Características clínico-demográficas de pacientes submetidos à cirurgia de revascularização do miocárdio e sua relação com a mortalidade. Rev Bras Cir Cardiovasc. 2012;27(1):52-60. 